\title{
Review Article \\ The Potential Therapeutic Effects of Artesunate on Stroke and Other Central Nervous System Diseases
}

\author{
Shilun Zuo, ${ }^{1}$ Qiang Li, ${ }^{1,2}$ Xin Liu, ${ }^{1}$ Hua Feng, ${ }^{1}$ and Yujie Chen ${ }^{1}$ \\ ${ }^{1}$ Department of Neurosurgery, Southwest Hospital, Third Military Medical University, Chongqing, China \\ ${ }^{2}$ Department of Neurobiology, College of Basic Medical Sciences, Third Military Medical University, Chongqing, China \\ Correspondence should be addressed to Hua Feng; fenghua8888@vip.163.com and Yujie Chen; yujiechen6886@foxmail.com
}

Received 2 August 2016; Accepted 20 November 2016

Academic Editor: Eduardo Gonzalez-Toledo

Copyright (c) 2016 Shilun Zuo et al. This is an open access article distributed under the Creative Commons Attribution License, which permits unrestricted use, distribution, and reproduction in any medium, provided the original work is properly cited.

\begin{abstract}
Artesunate is an important agent for cerebral malaria and all kinds of other severe malaria because it is highly efficient, lowly toxic, and well-tolerated. Loads of research pointed out that it had widespread pharmacological activities such as antiparasites, antitumor, anti-inflammation, antimicrobes activities. As we know, the occurrence and development of neurological disorders usually refer to intricate pathophysiologic mechanisms and multiple etiopathogenesis. Recent progress has also demonstrated that drugs with single mechanism and serious side-effects are not likely the candidates for treatment of the neurological disorders. Therefore, the pluripotent action of artesunate may result in it playing an important role in the prevention and treatment of these neurological disorders. This review provides an overview of primary pharmacological mechanism of artesunate and its potential therapeutic effects on neurological disorders. Meanwhile, we also briefly summarize the primary mechanisms of artemisinin and its derivatives. We hope that, with the evidence presented in this review, the effect of artesunate in prevention and curing for neurological disorders can be further explored and studied in the foreseeable future.
\end{abstract}

\section{Introduction}

Malaria has been a life-threatening disease for thousands of years in human history. With a variety of pharmacological activities, artemisinin has been widely considered as an essential antimalaria drug derived from a Chinese herb Qinghao. By the development of treating malaria, artemisinin overcame its alternatives by high efficiency and few side-effects [1]. Millions of patients who suffered from malaria were saved by artemisinin-based combination therapies (ACTs) every year [2]. In recognition of this great achievement, Youyou Tu won 2015 Nobel Prize in Physiology or Medicine, as one of the three contributing scientists to discovering artemisinin. Moreover, research has found that artemisinin not only possessed the antiparasite effect, but it also could have antitumor, anti-inflammation, and antimicrobes, and so forth effects. This therefore leads us to draw a conclusion that artemisinin is a pluripotent agent. Thus, to exploit its advantages, a large number of artemisinin derivatives have been synthesized according to different purposes. As one of the syntheses, artesunate was proved to be great promotion in the treatment of clinical disease.

Artesunate, the water-soluble semisynthetic derivative of artemisinin, has been a standard treatment for cerebral malaria and all kinds of other severe malaria [3]. The antimalarial effect of artesunate is commonly recognized as highly efficient, generally safe, and well-tolerated. In addition, numerous studies also pointed out that artesunate had widespread pharmacological activities, such as antiparasite, antitumor, antimicrobial, anti-inflammatory, antioxidant, impeding angiogenesis, protecting the $\mathrm{BBB}$, and immunoregulatory effect. And, more importantly, artesunate could be maintained as a higher concentration in brain [4], so that artesunate could be beneficial for treating diseases with rather intricate pathophysiologic mechanisms and multiple etiopathogenesis, such as neurological disorders.

As is known, the nervous system is one of the most complex and crucial systems in human body, resulting in considerable complicated neurological disorders. There are intricate pathophysiologic mechanisms and multiple etiopathogenesis 
involved in the occurrence and development of these disorders, whose clinic treatments are less effective by drugs with single mechanism and serious side-effects. In that case, importance should be attached to the pluripotent action of artesunate in study of the prevention and treatment of neurological disorders.

In this review, we initially depict the primary mechanisms of artemisinin and its derivatives in brief, followed by a thorough elaboration of the primary mechanisms of artesunate and its potential therapeutic effects on neurological disorders. Thereafter, we comment on past and current relevant basic studies and clinical trials. Moreover, we discuss possible research areas for further studying in future.

\section{Main Effects of Artemisinin and Its Derivatives}

2.1. Main Effects of Artemisinin. Since it was discovered back in 1972, researchers worldwide have carried out a large number of extensive and in-depth researches on artemisinin. The main efficacy of artemisinin they found was antiparasites, antitumor, anti-inflammation, antimicrobes, and so forth efficacy. In terms of antiparasites, artemisinin not only has a good effect on killing Plasmodium falciparum but also has promising antibabesial, antieimerial, antileishmanial, anticoccidian, antischistosomal, antitoxoplasmal, antitrypanosomal, and so forth activity. We are very surprised that artemisinin has such a wide range of antiparasites effects but even more amazed at its antitumor action. As early as the 1990s, artemisinin was found to have anticancer activity [5, 6]. A large number of studies have pointed out that artemisinin played the antitumor role in nervous system, respiratory system, digestive system, urinary system, reproductive system, blood system, and so forth. On the other hand, in the face of such powerful antitumor effect, we should note that these studies are mostly carried out in vitro and thus need to be confirmed in vivo. Another aspect that we need to be concerned about is the anti-inflammation action of artemisinin. Lu first reported Cordyceps and artemisinin could be used to prevent the recurrence of lupus nephritis and protect kidney function in 2002 [7]. Nonetheless, it has not attracted the attention of researchers until recent studies showed that artemisinin was very effective on autoimmune and allergic diseases. But we should also be aware that the anti-inflammatory effect of artemisinin still requires further confirmation due to the fact that studies are quite insufficient currently. Antimicrobial effect is another vital action of artemisinin. Some studies demonstrated that artemisinin worked well on eliminating some kinds of viruses, bacteria, and fungi. But these studies were also mostly conducted in vitro and need to be affirmed in vivo. There are other functions of artemisinin, containing the induction of neurite outgrowth, inhibiting vascular smooth muscle cells proliferation, and inducing apoptosis. Studies on these functions are mostly fragmented and need to be further verified. In general, the clinical application of artemisinin could be extensively broad, so that this domain is worth exploring.
2.2. Main Effects of Artemisinin Derivatives. As research develops, the deficiencies of artemisinin were gradually revealed. In order to overcome its shortcomings or to enhance some of its effect, a good many artemisinin derivatives have been synthesized according to different purposes, as in the case of dihydroartemisinin (DHA), artesunate, artemether, arteether, artelinic acid, artemiside, artemisone, artemisinic acid, artemisitene, artefenomel, arterolane, arteanuin B, SM735, SM905, SM933, SM934, SM1044, and so forth. Most of them have equivalent or even better antimalaria effect as artemisinin, such as DHA, artesunate, artelinic acid, artemether, arteether, arterolane, and so forth. Among them, artesunate and artelinic acid are water-soluble; SM735, SM905, SM933, and SM934 have shown strong immune regulatory functions. At present, DHA, artesunate, artemether, and arteether are used more widely in clinical practice. Their action is summarized accordingly in Table 1.

\section{Main Function and Advantage of Artesunate}

3.1. Advantage of Artesunate. As is known to us, artemisinin is not soluble in water; thereby it cannot be used for intramuscular or intravenous injection, which seriously restrains its effect in severe malaria. To overcome this shortcoming, artelinic acid and artesunate have been synthesized. Research reported that both of them could effectively kill Plasmodium falciparum, while, comparing with artesunate, artelinic acid had more embryotoxicity, neurotoxicity, nephrotoxicity, reticulocytopenia, vascular irritation, and anemia, and so forth. Artesunate was also defective owing to its ability of exterminating ring-stage parasites too rapidly which would result in delayed haemolysis [3]. Delayed haemolysis was frequent in patients (about 20\%) with severe malaria treated with intravenous artesunate $[8,9]$. Fortunately, the resulting anemia remained mild in $85 \%$ of cases [8]. And World Health Organization (WHO) guideline for treating malaria also indicated that, in individual parenteral artesunate doses between 1.75 and $4 \mathrm{mg} / \mathrm{kg}$, no toxicity has been observed [3]. Besides, the price is cheap relatively. Consequently, artesunate was deemed to be the most striking in all the artemisinin derivatives and also the only clinical application of watersoluble derivatives of artemisinin. WHO has strongly recommended intramuscular or intravenous artesunate as the first-line treatment for cerebral malaria and all kinds of other severe malaria [3].

Another noteworthy advantage is that artesunate could be maintained as a higher concentration in brain. It is suggested even if the drug levels of artesunate dropped significantly in all tissues at $1 \mathrm{~h}$, the proportion in brain, fat, intestine, and serum is still high and with very slight neurotoxicity [4]. This implied that artesunate might have superb advantages in the treatment of nervous system diseases.

3.2. Main Function of Artesunate. Artesunate is prepared from DHA by reacting it with succinic acid anhydride in basic medium. The same as artemisinin, it has four main functions: antiparasites, antitumor, anti-inflammation, antimicrobes functions. Of these four, antiparasitic function plays the most classic and important role for artesunate, 


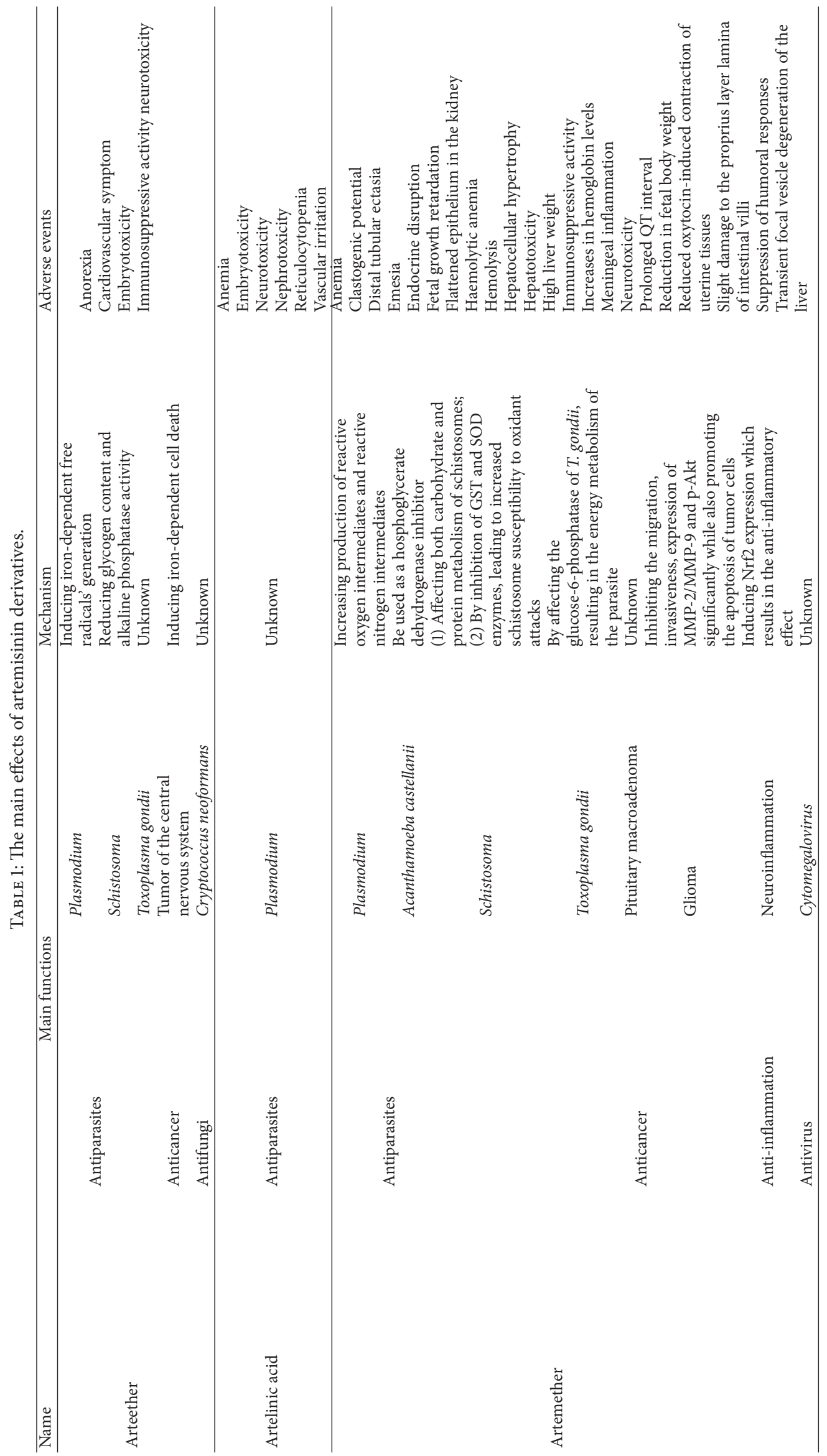




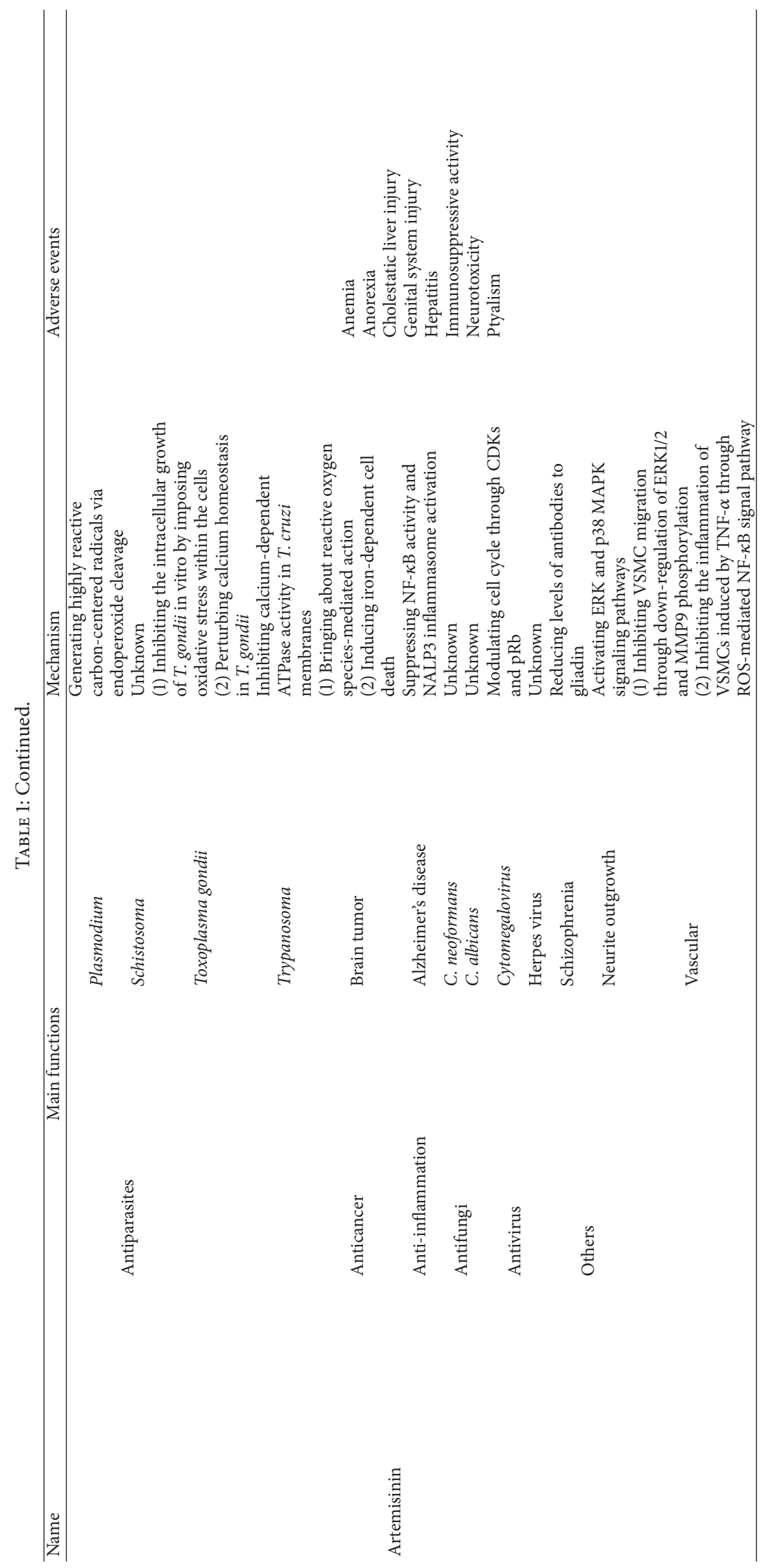



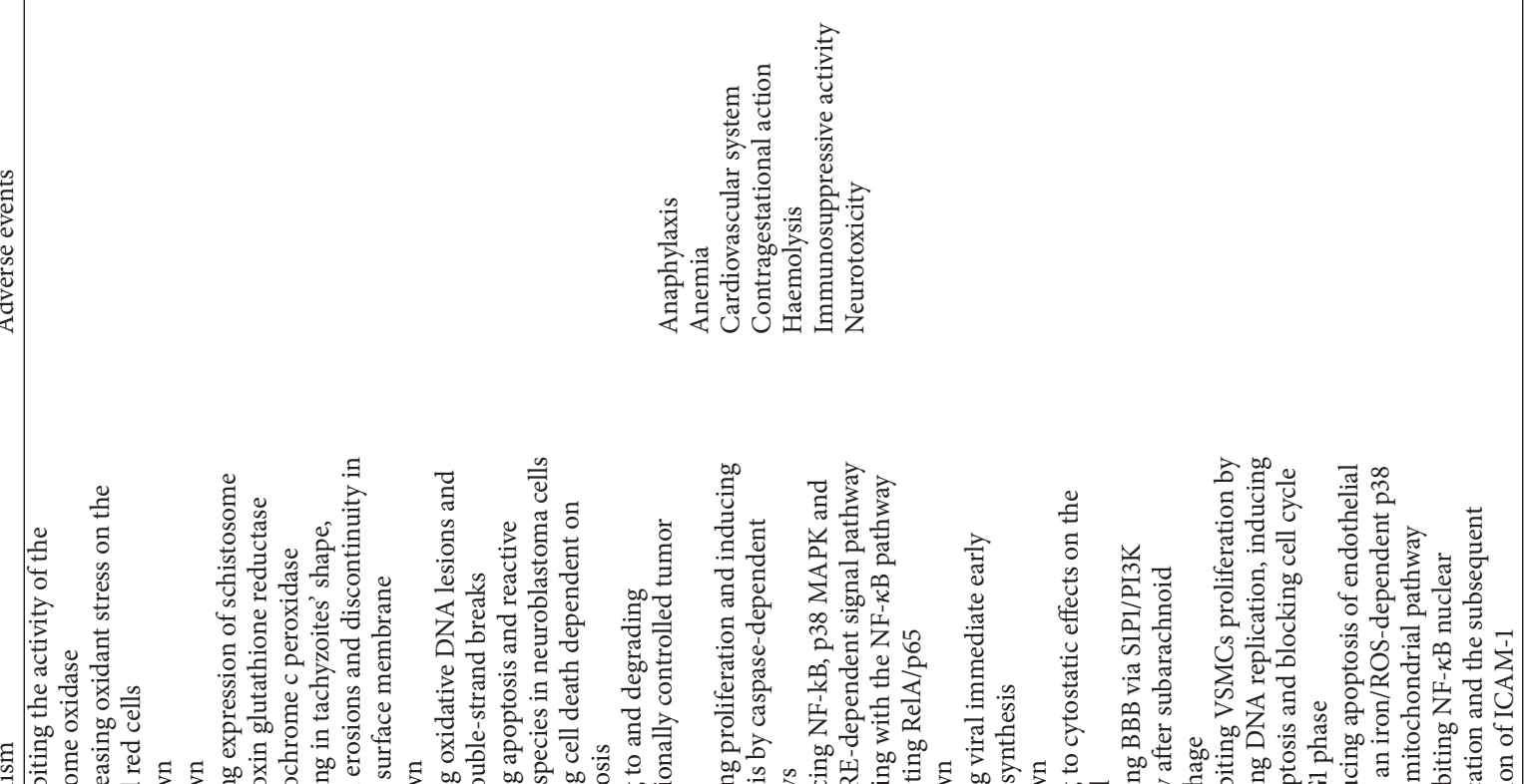

: :

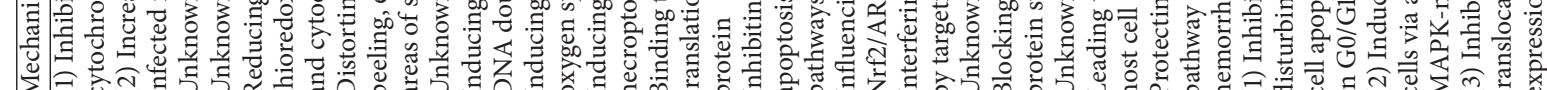

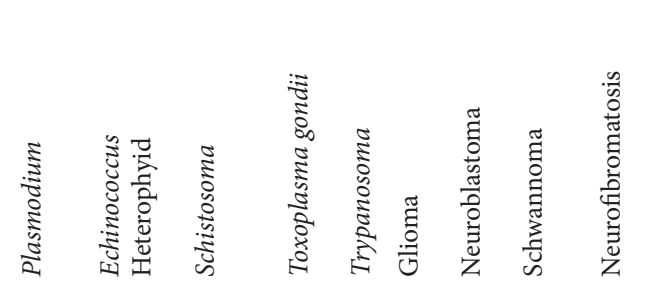

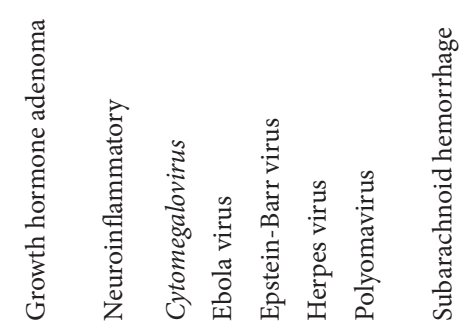

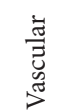

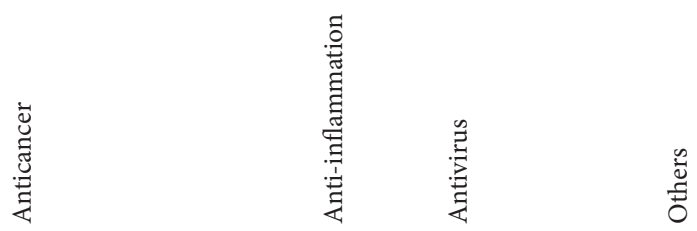




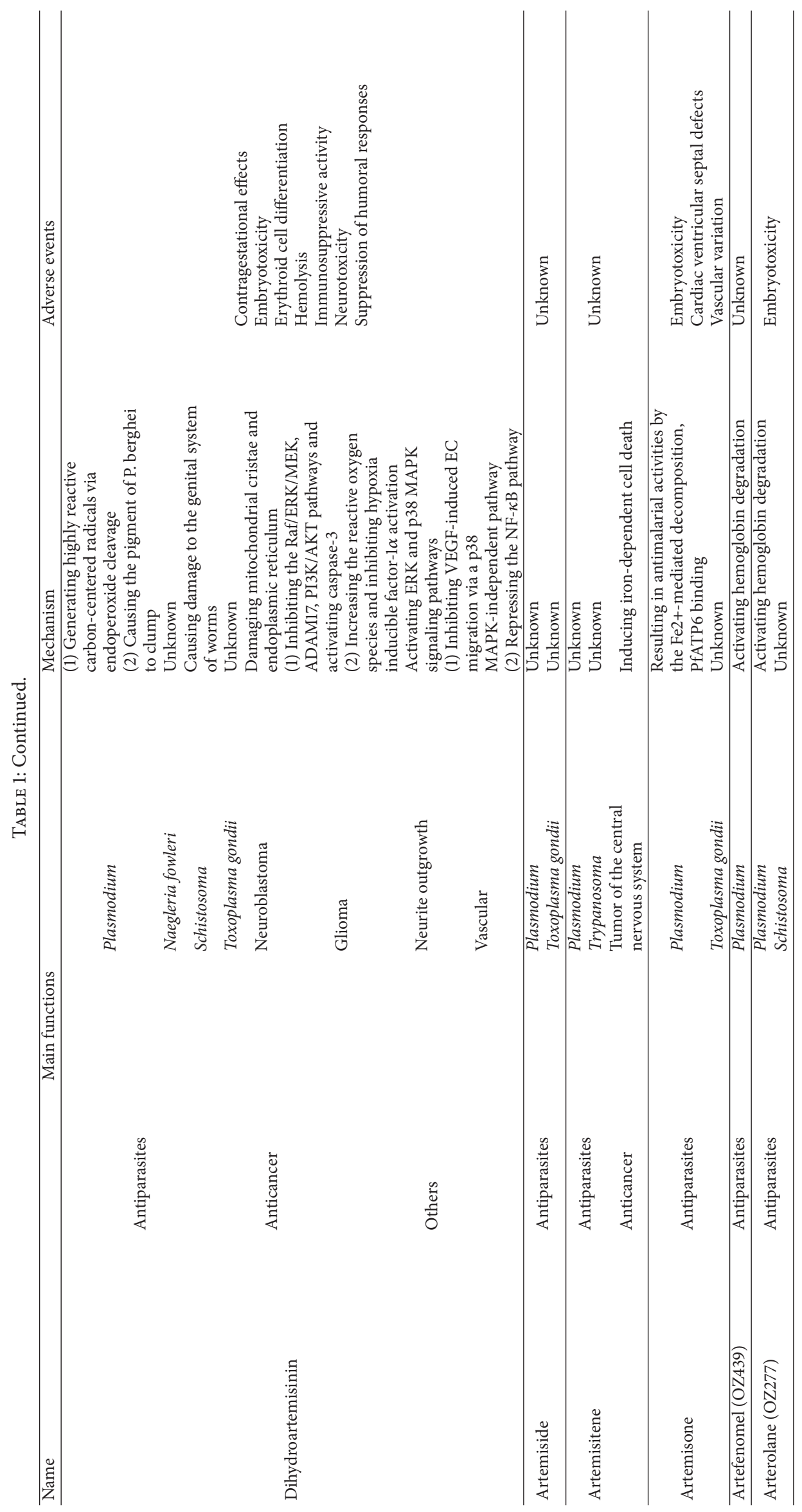




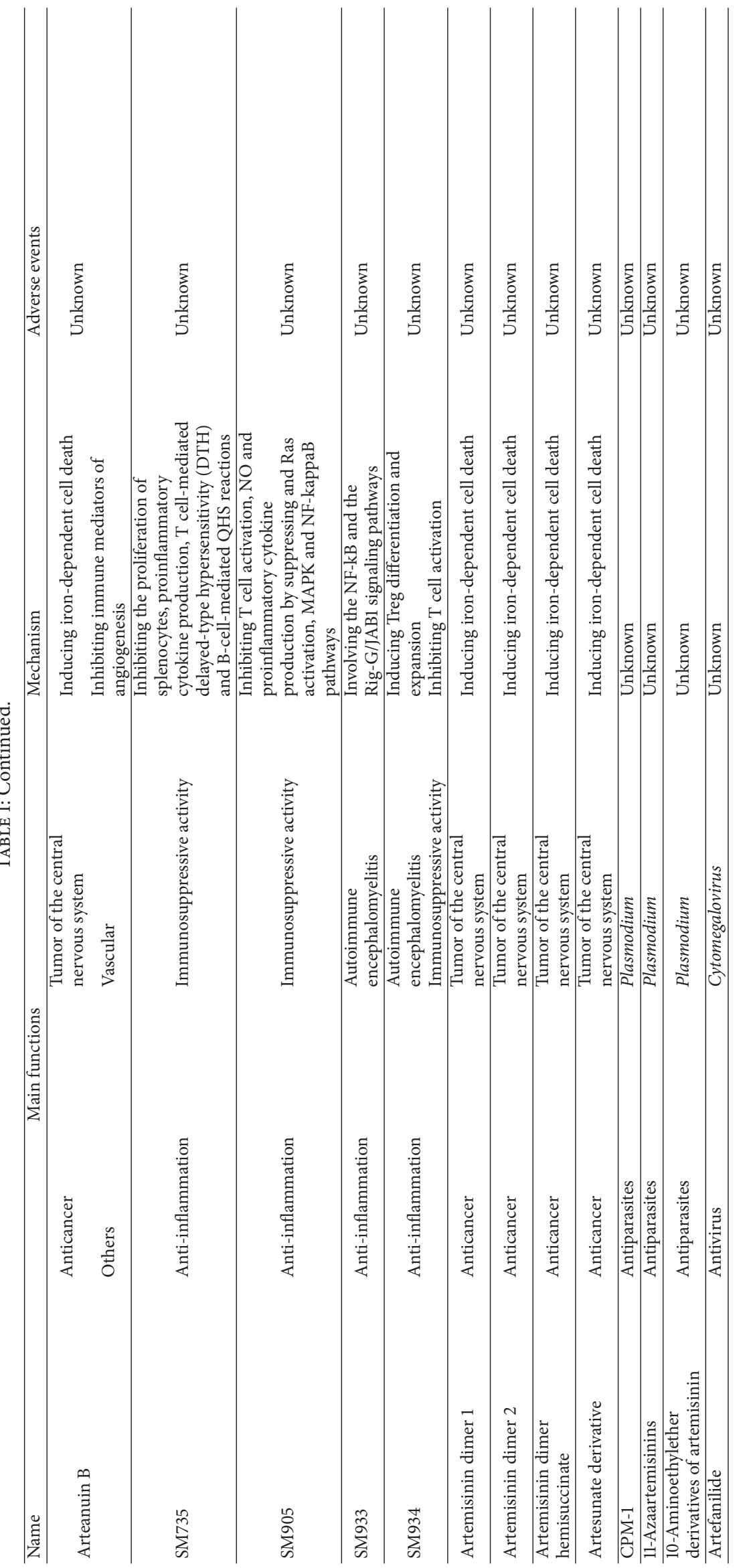


due to the effect of killing Plasmodium falciparum. Malaria remains an important cause of illness and death in children and adults in countries in which it is endemic. And its control requires an integrated approach, including prevention and prompt treatment with effective antimalarial agents. The third edition of guidelines for the treatment of malaria especially emphasized that treating adults and children with severe malaria (including infants, pregnant women in all trimesters, and lactating women) with intravenous or intramuscular artesunate was a recommended treatment, which fully illustrated its importance on antimalaria. The antimalarial mechanism of artesunate works primarily by means of completely inhibiting the activity of the cytochrome oxidase in the Plasmodium and promoting the generation of active oxygen species in erythrocytes $[10,11]$. Likewise, schistosomiasis is considered by WHO as the second most socioeconomically devastating parasitic disease (after malaria). More than 61.6 million people have been reported for being treated for schistosomiasis in 2014 (721, 722). Massive studies both in vivo and in vitro confirmed that artesunate is credibly effective on $S$. japonicum and S. mansoni. These findings allow us to have a new choice in the treatment of schistosomiasis. Artesunate is also effective on other parasites, like Fasciola hepatica, Clonorchis sinensis, Babesia, Leishmaniasis and Toxoplasma gondii, and so forth (Table 1), which fully illustrated that artesunate had broad antiparasitic effects.

Cancer is one of the leading causes of death in the world. Research indicated that artesunate could inhibit or even kill as many as 32 kinds of tumor cells, for instance, glioblastoma, esophageal cancer, breast cancer, lung cancer, hepatocellular carcinoma, prostate cancer, ovarian cancer, leukemia and myeloma (Table 1). As for why artesunate has such wide and highly effective antitumor capabilities, recent studies suggested artesunate could induce ROS response in tumor cells, specifically the oxidative DNA lesions and doublestrand breaks $[12,13]$; and it could increase the apoptosis of tumor cells by downregulation of the expression of PCNA and $\mathrm{Bcl}-2$ genes and upregulation of the expression of $\mathrm{Bax}$ gene as well [14]; what is more, it also could enhance the radiosensitivity by reducing the expression of the inhibitor of apoptosis protein surviving [15] and so forth. In addition, recent research has shown that artesunate could induce ferroptosis in tumor cells by increasing the expression of iron-related genes [16]. This finding represented an attractive strategy for cancer therapy in the future. In spite of how exciting these findings are, we should note that these achievements, mainly derived from in vitro experiments, need to be confirmed further in vivo.

Anti-inflammation is the third main effect of artesunate. Evidence showed that it had a good effect on autoimmune and allergic diseases, such as allergic asthma, arthritis, dermatitis, hepatic fibrosis, pulmonary fibrosis, and myasthenia gravis (Table 1). Current thinking suggested that the anti-inflammatory mechanism of artesunate was mainly to inhibit the production of autoantibodies, proinflammatory cytokines and inflammatory cytokines, and so forth. Nevertheless, despite the successful application in many inflammatory disease models, study on anti-inflammatory efficacy of artesunate is quite limited and further research is still required.
The last but not least major role of artesunate is antimicrobes role. At present, the drug resistance of microorganisms is becoming more and more severe. To solve this problem, we need to find synergistic effect between antibiotics and nonantibiotic drugs. In that case, we are delighted with the capability of artesunate of providing synergistic effects with some antibiotics. Research reported artesunate could enhance the anti-MRSA (methicillin-resistant Staphylococcus aureus) activity of oxacillin [17]. Additionally, it also could kill Saccharomyces cerevisiae [18] and inhibit the replication of some kind of virus, such as Cytomegalovirus, Ebola virus, Epstein-Barr virus, polyomavirus, hepatitis B virus, and herpes virus (Table 1). And the effect of inhibiting the replication of Cytomegalovirus has been confirmed in clinical trials. As for other kinds of virus, this needs to be further studied.

Artesunate also has effect on other diseases, such as cardiovascular disease, hyperlipidemia, diabetes, lung injury, and hypertrophic scar (Table 1). These studies, however, are not sufficient and need to be further confirmed. To sum up, artesunate has widespread pharmacological activities, requiring further exploration and study.

\section{Effect of Artesunate on Central Nervous System Diseases}

4.1. Central Nervous System Infections. For years, a number of efficient antibiotics have been widely used in clinical practice, until the issue of drug resistance arises. Consequently, the antimicrobes function of artesunate got back to our sight. Antimicrobes role is one of the primary roles of artesunate; many pathogens that cause the infection of the CNS are in the range of its actions. For instance, Staphylococcus aureus is the pathogen of epidural abscess, subdural abscess, purulent meningitis, brain abscess and intraspinal abscess, and so forth. Research reported artesunate could enhance the anti-MRSA activity of oxacillin [17]; when combined with ampicillin sodium-sulbactam sodium, it could further increase survival rate of mice challenged with S. aureus [19]. Virus is another important cause of infection in CNS. Cytomegalovirus, herpes simplex virus, and Epstein-Barr (EB) virus can lead to the occurrence of viral encephalitis. And the human JC polyomavirus is a leading cause of the fatal demyelinating disease progressive multifocal leukoencephalopathy (PML). Studies have shown that artesunate could obviously inhibit the replication of the Cytomegalovirus, herpes simplex virus, polyoma virus, and EB virus in vitro (Table 1). Furthermore, animal experiments confirmed that artesunate combined with valacyclovir had a good effect on mice with herpes simplex virus encephalitis [20]. Fungal infections of the CNS are rare, and the main pathogenic fungi were Candida, Cryptococcus, Mucor, and Actinomycetes. There are few studies on fungicidal efficacy of artesunate, conveying artesunate has distinctly killing effect on Saccharomyces cerevisiae, while artemisinin has a good killing effect on Aspergillus fumigatus, C. neoformans, and $C$. albicans in vitro (Table 1). Whether artesunate has a similar effect needs further study to be confirmed. 
The CNS parasitic disease, caused by parasites invading the CNS, is part of systemic parasitic disease. The main pathogens of the CNS parasitic diseases are cysticercus cellulosae, Paragonimus westermani, Schistosoma, Echinococcus and Toxoplasma gondii, and so forth. As the disease is part of systemic parasitosis, the researches for the CNS parasitic infection are few. Research indicated that artesunate had evident inhibitory effect on Toxoplasma gondii in vitro and in vivo [21]. Moreover, artesunate-DHA combination could substantially reduce the size of the cerebral cysts of Toxoplasma gondii in mice after only 5 days of treatment [22]. In 1983, Le et al. first reported that artesunate was demonstrated to exert antischistosomal effect in experimental animals infected with Schistosoma japonicum and had no severe side-effects [23]. Subsequently, the antischistosomiasis effect of artesunate was further confirmed in different animal models, such as mice, rabbits, dogs, and even human. Present study suggests that the antischistosomiasis effect of combining artesunate-praziquantel is superior to each of them alone [24]. Unfortunately, there is no research report on nervous system of schistosomiasis. Echinococcosis, also called hydatid disease, is a parasitic disease of tapeworms of the Echinococcus type. The disease is widely distributed around the world and causes great loss of life and property every year. Cerebral echinococcosis and intraspinal hydatid disease are rare but seriously affect the human health. One piece of research pointed out that artesunate could effectively kill Echinococcus multilocularis and Echinococcus granulosus in vitro but had no effect in vivo [25]. Cysticercus cellulosae and Paragonimus westermani are important pathogens in the CNS parasitic diseases; however, no study mentioned the effects of artemisinin and its derivatives on both of them.

In short, the effects of artesunate for treatment of the CNS infections and parasites are affirmative and worthy of being studied deeply.

4.2. Vascular Diseases of the Brain and Spinal Cord. Vascular diseases of brain and spinal cord mainly include subarachnoid hemorrhage (SAH), intracerebral hemorrhage (ICH), ischemic cerebrovascular disease (ICD), intracranial aneurysm, intracranial vascular malformation, carotidcavernous fistula, moyamoya disease, and spinal vascular malformation. Establishment of animal models of $\mathrm{SAH}$, $\mathrm{ICH}$, and ischemic cerebrovascular disease is much easier than other diseases; therefore, numerous studies have mostly concentrated on these three diseases at present.

4.2.1. Subarachnoid Hemorrhage. SAH is a devastating cerebrovascular disease with complex mechanisms, closely linked with high morbidity and fatality rate at relatively young age and leading to a great loss of the economy and society. Artesunate has wide impact on animal body system, one of which is protecting BBB after SAH. Our study demonstrated that artesunate could preserve blood-brain barrier (BBB) integrity and improve neurological outcome after SAH, possibly through activating $\mathrm{S}_{1} \mathrm{P}_{1}$, enhancing $\mathrm{PI} 3 \mathrm{~K}$ activation, stabilizing $\beta$-catenin via GSK-3 $\beta$ inhibition, and then effectively raising the expression of Claudin 3 and Claudin 5 [26].
In addition, artesunate could attenuate the generation of pro inflammatory mediators in BV2 microglial cells by influencing NF-kappaB, p38 MAPK, and Nrf2/ARE-dependent signal pathway $[27,28]$. As is well known, microglial cells play an important role in the pathogenesis of SAH. Inhibition of microglia activation could alleviate the early brain injury (EBI) after SAH. Furthermore, recent research demonstrated that intraparenchymal activation of accumulated resident microglia, between days 4 and 28 after SAH, was accountable for neuronal cell death [29]. Therefore, artesunate probably has a protective effect on secondary brain injury after SAH. Another noticeable therapeutic effect is that artesunate could inhibit vascular smooth muscle cells (VSMC) proliferation by disturbing DNA replication, blocking cell cycle in G0/G1 phase, and inducing cell apoptosis [30]. Early studies had shown that VSMC proliferation and subsequent vessel wall thickening contributed to the syndrome of delayed cerebral vasospasm after SAH [31]. Thus, we have reason to believe that artesunate could prevent damage from delayed brain injury (DBI). Mounting evidence indicates that the leukocyte exerts a crucial effect in the pathophysiology of SAH. Elevated leukocyte count has been reported to increase the risk of experiencing symptoms of vasospasm, and it was closely associated with a $90 \%$ rate of poor outcomes [32]. Leukocytes adherence and accumulation in brain vessel, an important feature of the late-stage of cerebral malaria, could be rapidly decreased by artesunate [33]. Such studies said that artesunate could not only regulate the function of leukocyte but also destroy it [34-36]. So, the effect of artesunate on leukocytes after SAH is worthy of further study. Adhesion molecule, an important mediator of inflammation after stroke, mediates endothelial capture, adhesion, extravasation of leukocytes, and recruitment to the site of injury [32]. Intercellular adhesion molecule-1 (ICAM-1), an important adhesion molecule, could help leukocytes binding to endothelial cells and then transmigrating into brain tissue [37]. During the early and late period after $\mathrm{SAH}$, the level of ICAM-1 was significantly elevated in the serum of patients [38]. Monoclonal antibodies against ICAM-1 could attenuate cerebral vasospasm by $22 \%$ after SAH in rabbits [39]. As anti-ICAM-1 antibody, artesunate could inhibit the expression of ICAM1 in order to resist the $P$. berghei-induced inflammatory response [40]. It implied that artesunate probably also had the effect of inhibiting the expression of ICAM-1 after SAH. Aside from neurological injuries, $\mathrm{SAH}$ has been associated with nonneurologic medical complications, and pulmonary complications are one of them. Pulmonary complications are the most frequent extracerebral cause of death after SAH [41, 42], which include pneumonia, aspiration, and neurogenic pulmonary edema [43]. Pulmonary complications in SAH patients occurred under a few mechanisms, and one of them is SAH induced expression of the proinflammatory mediators in the lung [44]. We know that artesunate possesses antiinflammatory and antioxidative properties. Relevant research indicated that artesunate, through controlling the expression of both proinflammatory mediators and prooxidants, could significantly prevent the lung injury of rodents induced by ovalbumin, cigarette smoke, and paraquat [45-47]. This 
research implied that artesunate might have therapeutic potential for treating pulmonary complications after SAH.

Shortly, artesunate has significant therapeutic effects on animal model of SAH, and the studies on the mechanisms have achieved some progress, but there are still many problems that need to be deeply investigated.

4.2.2. Intracerebral Hemorrhage. $\mathrm{ICH}$ is a stroke subtype closely linked with high mortality (about $40.4 \%$ at 1 month), and even the surviving patients frequently have serious neurological damage [48]. More than primary brain injury, secondary injury after ICH needs drug treatment. Secondary injury could be caused by a cascade of events initiated by the primary injury, such as thrombin, inflammation, complement, hemoglobin, iron, cell-death pathways, endogenous defence mechanisms, and free radicals [49]. The effects of artesunate for treating the $\mathrm{ICH}$ have not been reported at present. However, numerous studies of artesunate have reminded us that it might play an important role in the secondary injury after ICH. For instance, inflammatory reaction was the basic factor causing the secondary injury after ICH, which was mainly concerned with activation of resident microglia, an influx of leucocytes into the brain, and production of inflammatory mediators, and so forth [49]. One of the main functions of artesunate is antiinflammation. Mentioned in the preceding paragraphs, artesunate could attenuate the generation of proinflammatory mediators in BV2 microglial cells and prevent leukocytes from transmigrating into brain tissue through inhibiting the expression of ICAM-1 on endothelial cells. In addition, another important factor causing the secondary injury after $\mathrm{ICH}$ was that endogenous defence mechanisms were barely strong enough to resist the oxidative stress. Nuclear factor erythroid 2-related factor 2 (Nrf2), a transcription factor that responds to oxidative stress, might be a key regulatory factor of endogenous defence mechanisms after ICH [50]. Studies found that artesunate was a potential activator of the Nrf2/ARE-dependent signal pathway, which could attenuate LPS-induced inflammatory responses in microglial BV2 cells [28]; and it significantly enhanced the expression of $\mathrm{Nrf} 2$ in lung tissues from ovalbumin-challenged mice and in TNF-alpha-stimulated human bronchial epithelial cells [46]. Autophagy is another contributing factor to the secondary injury after ICH. Research indicated that many substances could activate autophagy which caused secondary injury in the brain after ICH [51-53]. We are glad to see that the suppressed autophagy effect of artesunate has been confirmed in in vitro experiment [54]. In a word, there are reasons to believe that artesunate would exert effect in the secondary injury after ICH.

Confusingly, artesunate also could enhance the formation of reactive oxygen species and then induce DNA doublestrand breaks in P. falciparum and killing cancer cells [55, 56]. About autophagy, other researches proposed that artesunate, through upregulating the expression of Beclin1 and stimulating the aggregation of LC3, induced autophagy in tumor cells and then exerted the antitumor effects [57]. Many contradictions about the effects of artesunate exist. Therefore, it needs further experimental studies to confirm the effect in the secondary injury after $\mathrm{ICH}$.
4.2.3. Ischemic Cerebrovascular Disease. Ischemic cerebrovascular disease (ICD), the most common and frequent cerebrovascular disease, is one of the main causes of morbidity and mortality all over the world. So far, no research on the effects of artesunate for treatment of the ICD has been reported. We suspect that artesunate would exert effect in the ICD because it has a wide spectrum of pharmacological effects. For example, cerebral ischemic injury, an important clinical appearance of ICD, is a condition of complex pathology that includes several inflammatory events, such as aggregation of inflammatory cells, activation of immune cell responses, and upregulation of cytokines [58]. Artesunate, as mentioned earlier, could inhibit the aggregation of inflammatory cells and upregulation of cytokines. Toll-like receptors (TLRs), as an important mediator of cerebral ischemic injury, have played an important role in the initial activation of immune cell responses. Studies found that artesunate could significantly reduce the expressions of TLR4 and myeloid differentiation factor 88 (MyD88) in lipopolysaccharidestimulated proinflammatory responses and experimental hepatic fibrosis, which serves as the underline mechanism for the anti-inflammation effect of artesunate [59-61]. So, artesunate probably has the effect of inhibiting the activation of immune cell responses in cerebral ischemic injury. In addition, ischemia/reperfusion injury is most likely to occur after intravenous thrombolysis which is the standard of care for patients with acute ischemic stroke. Previous studies found that antioxidation and scavenging free radicals could alleviate the ischemia/reperfusion injury in rats [62]. As noted above, artesunate has the effect of antioxidation and scavenging free radicals in microglial BV2 cells. Therefore, artesunate probably has the therapeutical effect of the cerebral ischemia/reperfusion injury after ICD. Hemorrhagic transformation (HT) is a frequent asymptomatic event after ICD, especially in the reperfusion after acute ischemic stroke [63]. Hamann and his colleagues suggested that compromised microvascular basal lamina integrity might be a potential precursor of HT [64]. The activation of proteolytic enzymes, especially matrix metalloproteinases 9 (MMP-9), was thought to further be contributed to dysfunction of the basal lamina [65]. Artesunate could antagonize the expression of MMP-9 in the haemozoin-induced human monocytic cells and the hepatic tissue of cirrhotic rats induced by bovine serum albumin $[66,67]$. Accordingly, artesunate may prevent the occurrence of HT after acute ischemic stroke. Diabetes and ICD often arise simultaneously. Patients with diabetes have more than double the risk of ICD after correction for other risk factors, relative to individuals without diabetes [68]. Yu et al. newly found that artesunate could elicit a protective effect on pancreatic beta-cells exposed to IL-1 $\beta$ by stimulating SIRT1 expression, which resulted in the decrease of NF-kB activity, iNOS expression, and NO production [69]. Hence, ART might be an effective drug for diabetes after ICD. Intracranial atherosclerosis, one of the leading causes of ICD, is associated with an increased risk for recurrent ICD [70]. Hypercholesterolemia was an independent risk factor for intracranial atherosclerosis. One finding was that artesunate $(25 \mathrm{mg} / \mathrm{kg})$ could significantly decrease the plasma 
cholesterol and triglyceride in rabbits [71]. This implied the preventative effect of artesunate to ICD.

In general, artesunate probably has effect on prevention and treatment of ICD. But the roles in some aspects that artesunate play require further confirmation. For instance, as mentioned above, $25 \mathrm{mg} / \mathrm{kg}$ artesunate on hypercholesterolemia was effective in rabbits [71]. Meanwhile some other researches on artesunate pointed out $50 \mathrm{mg} / \mathrm{kg}$ had no effect on the levels of cholesterol in rats [72]. Moreover, artesunate was not very efficient on inhibition of the expression of MMP-9. The study found that artesunate could only reduce the expression of MMP-9 in the haemozoin-induced human monocytic cells by $25 \%$, while DHA could reduce that by $50 \%$ [67]. Thus, it is needed to validate the effect of artesunate on prevention and treatment of ICD with experimental data.

4.3. Nervous Tissue Tumors. The nervous tissue tumors can be categorized as primary or metastatic. The incidence and prevalence of primary nervous tissue tumors outweigh metastatic nervous tissue tumors by $1: 4$ [73].

4.3.1. Primary Nervous Tissue Tumors. The primary nervous tissue tumors include central nervous system tumors and peripheral nervous system tumors, most of whose underlying causes remain unclear. Currently, treatments for the nervous tissue tumors could be separated into three categories: blasting it with radiation, surgically removing a tumor, and using drugs to kill the cancerous cells. The toxicity and various side-effects of the drugs and the uncertain outcome of chemotherapy in brain tumors put this treatment further down the line of treatment options with surgery and radiation therapy preferred. But treating tumor with chemotherapeutic drugs is always the hot topic of research. In addition to high potential and low toxicity, artesunate could also pass through the blood-brain barrier and be maintained as higher concentration in the nervous system [4]. These characteristics of artesunate were paid attention to by oncology researchers and lots of researches were carried out. Glioblastoma is the most common and aggressive cancer that starts within the brain. Research suggested that artesunate could induce oxidative DNA lesions and sustain double-strand breaks and finally lead to human glioblastoma cells death [12]. Moreover, several studies have pointed out that a combination of artesunate and temozolomide or OSI-774 might result in increased cytotoxicity in glioblastoma cells [74-76]. What is more significant is that artesunate could selectively decline survivin that contributes to radiosensitization of glioblastoma cells by an increased induction of apoptosis, cell cycle arrest, and a hampered DNA damage response [15]. Neuroblastoma is a type of pediatric tumor of neural crest origin with heterogeneous phenotypes [77]. Currently, there is no adequate therapy for refractory neuroblastoma and the mechanisms underlying its chemoresistance are poorly understood. As early as 2010, Michaelis et al. pointed out that neuroblastoma represented an artesunate-sensitive cancer entity and that artesunate was also effective in chemoresistant neuroblastoma cells [78]. The growth hormone (GH) adenoma could secrete excessive $\mathrm{GH}$ which causes acromegaly. Not only could artesunate inhibit proliferation and induce apoptosis in GH3 cells by caspase-dependent pathways, but it could also restrain GH synthesis and secretion [79]. In conclusion, artesunate has apparent therapeutic effects on some central nervous system tumors.

Schwannoma is a benign nerve sheath tumor composed of Schwann cells and the most common tumor of peripheral nerves. Study indicated that artesunate effectively induced cell death in RT4 schwannoma cells and human primary schwannoma cells by suppressing autophagy and promoting necroptosis [80]. Neurofibromatosis type 1 (NF1) is an autosomal dominant disease that predisposes individuals to develop benign neurofibromas and malignant peripheral nerve sheath tumors (MPNSTs). The research suggested that a positive feedback loop between TCTP and mTOR contributed to NF1associated tumor formation [81]. Artesunate, which binds to and degrades TCTP, significantly suppressed the viability of MPNST cells but not normal Schwann cells [81]. Moreover, combinational usage of artesunate and rapamycin enhances the cytotoxic effect on MPNST cells [81]. This demonstrates that artesunate has remarkable therapeutic effect against peripheral nerve sheath tumor.

4.3.2. Metastatic Nervous Tissue Tumors. Lung and breast carcinoma make up the majority of metastatic tumors. There are a large number of studies on the role of artesunate in the treatment of lung cancer. For example, research demonstrated that artesunate could induce Bak-mediated caspase-independent intrinsic apoptosis in both ASTC-a1 and A549 lung adenocarcinoma cell lines [82]. And artesunate combined with NP (a chemotherapy regimen of vinorelbine and cisplatin) could elevate the short-term survival rate and prolong the time to progression of patients with advanced non-small cell lung cancer without extra sideeffects [83]. These findings suggested that artesunate had potent therapeutic effect for lung cancer. Notably, artesunate could also significantly impair matrigel invasion of A549 cells through inhibiting urokinase-type plasminogen activator $(\mathrm{u}-$ PA) activity and the expression of u-PA, ICAM-1, MMP-2, MMP-7 and MMP-9, and so forth [84, 85]. This reminds us that artesunate possibly could stop the brain metastases of lung cancer. Artesunate could also inhibit the proliferation and differentiation of breast carcinoma through activating programmed cell death in vivo and in vitro $[55,86,87]$. When used in combination with lenalidomide or camptothecin, artesunate could induce higher apoptosis rates in breast carcinoma cells $[88,89]$. These researches imply that artesunate may have curative effect for the brain metastases of breast carcinoma.

To sum it up, artesunate has obvious curative effects for some primary and metastatic nervous tissue tumors with various mechanisms. However, we must note that the effect of artesunate against nervous tissue tumors mainly come from in vitro experiments, which needs to be certified further in vivo.

4.4. Neurological Autoimmune Diseases and Neurodegenerative Disorders. Autoimmune diseases stemming from an abnormal immune response of the body resist substances and tissues normally present in the body. Neurological 
autoimmune diseases are part of the autoimmune diseases. Artesunate exhibited potent immunosuppressive activity in several autoimmune disease models. For example, the research indicated that artesunate could prevent arthritis development in young $\mathrm{K} / \mathrm{BxN}$ mice by inhibiting germinal center formation and production of autoantibodies [90]. And, also, artesunate could inhibit the progression of disease and reverse the pathologic lesion of lupus nephritis in MRL/lpr mice through decreasing the level of MCP-1 and BAFF [91]. However, it is surprising that, during the treatment of neurological autoimmune diseases, artesunate has not been reported. Notably, a case indicated that a woman that suffered from mixed malaria infection, while recovering by artesunate and doxycycline therapy, ended up with developing acute disseminated encephalomyelitis (ADEM) [92]. Does this mean artesunate has no effect on ADEM and even neurological autoimmune diseases? This is the issue worth exploring and studying in the future.

Neurodegenerative disorders, mainly including amyotrophic lateral sclerosis, Parkinson's disease, Alzheimer's disease, and Huntington's disease, occur as a result of neurodegenerative processes. There has been no report about the therapeutic effect of artesunate on neurodegenerative disorders. However, study clearly demonstrated that artemisinin had protective effects on Alzheimer's disease pathology through suppressing NF- $\kappa$ B activity and NALP3 inflammasome activation in APPswe/PS1dE9 transgenic mice [93]. As the watersoluble derivatives of artemisinin, the protective effects of artesunate on Alzheimer's disease or even neurodegenerative disorders merit in-depth studies.

4.5. Epilepsy. Epilepsy is a common disease in nervous system. Epilepsy is also an important sign of severe malaria, particularly in children. For instance, study showed that epilepsy was present at $25.6 \%$ of 261 malaria patients from 3 tropical clinics in South Sudan [94]. Artesunate could effectively inhibit seizure caused by severe malaria. Research suggested that prevalence of seizure in African children with severe malaria after 6 hours of admission was $10.1 \%$ in the quinine group and $8.3 \%$ in the artesunate group $(p=0.0199)$ [95]. However, the cause of most patients of epilepsy was unclear, although some developed epilepsy as a result of traumatic brain injury, brain tumors, stroke, intracranial infections, birth defects, and so forth. Whether antiepileptic effect of artesunate is the optimal therapy for other origins of epilepsy requires further confirmation. Another issue that we need to be concerned about is that animal experiments could not completely simulate human epilepsy, such as the antiepileptic properties of quinine which only worked in animals, not in human [96]. So, a discreet selection of animal models is needed to confirm the antiepileptic effect of artesunate.

4.6. Traumatic Brain Injury and Spinal Cord Injury. Traumatic brain injury (TBI), which occurs when an external force traumatically injures the brain, is a leading cause of mortality and is a major public health issue in children and young adults. No research has investigated the treatment effect of artesunate on TBI so far. Various findings suggested indirectly that artesunate probably played a role in treatment of TBI. For example, one of the primary concerns was controlling raised intracranial pressure (ICP) after TBI, since high ICP could reduce the level of blood-oxygen in brain and cause deadly brain herniation [97]. As stated above, artesunate could preserve $\mathrm{BBB}$ integrity, reduce brain water content, and then improve neurological outcome after SAH. Thus, the role of artesunate played may contribute to the reduction of ICP after TBI. In addition, artesunate could also inhibit the expression of ICAM-1 on endothelial cells, attenuate the generation of proinflammatory mediators, and activate the Nrf2/ARE-dependent signal pathway in microglia. These studies reminded us that artesunate might play an important role in the secondary injury after TBI. To summarize, there is reason for optimism, but we must confirm the therapeutic effect of artesunate on TBI by experiment.

Spinal cord injury (SCI) is the damage to the spinal cord that causes partial or total loss of muscle function, sensation, and autonomic nerve function below the level of the lesion, either temporary or permanent. So far, we have found no report about artesunate on SCI. As described above, artesunate has a powerful anti-inflammatory and antioxidant effect, so we have reason to believe that it has a certain therapeutic effect on SCI.

\section{Perspective and Conclusion}

As is widely known, the occurrence and development of neurological disorders can be extremely complicated. A number of pathogenic mechanisms are involved in different pathological progress of neurological disorders. Several pathogenic mechanisms would interact with each other even at the same period of one neurological disorder, which is becoming a huge obstacle during treatment of diseases. Agents with more than one protective effect are attractive as potential therapeutic drugs of neurological disorders. Artesunate has a powerful protective effect, such as antineuroinflammatory, antiparasite, antitumor, antimicrobial, antioxidant, protecting the BBB, and immunoregulatory effect. Therefore, we have reasons to believe that it also has certain therapeutic effects on other neurological disorders. Moreover, artesunate not only could dissolve in water and be maintained as a high concentration in the CNS but also has fewer side-effects and can be fairly cheap. So if any therapeutic effect of artesunate on other neurological disorders can be discovered, it will be of benefit both socially and economically. In a word, artesunate is worthy of further study in neurological disorders.

A single medication only has limited therapeutic effects; therefore, combined medicine has more advantages for preventing or reversing occurrence and development of diseases. A large number of studies implied that when combined with other drugs, artesunate could enhance therapeutic effects on parasites, microbes, tumors, and so forth. Thus, we should actively explore the combined therapeutic effect of artesunate on neurological disorders.

Biochemical targets of artesunate are another subject that deserves greater attention. There is no consensus on biochemical targets of artesunate at present. Excitedly, re cent research has shown that DHA could specifically target 
PfPI3K [98]. However, whether the PfPI3K is also the biochemical target of artesunate remains unknown and requires further research. Elsewhere, the mechanisms of artesunate on some signal pathway need to be further elucidated. For instance, our evidences showed that artesunate could activate the PI3K/Akt pathway, but some research found the opposite $[99,100]$.

In conclusion, artesunate, as a pluripotent agent, needs to be further explored and studied on the effect of prevention and treatment of neurological disorders.

\section{Competing Interests}

The authors declare that there is no conflict of interests regarding the publication of this paper.

\section{Acknowledgments}

This study was funded by the National Natural Science Foundation of China (no. 81501002 to Yujie Chen, no. 81220108009 to Hua Feng) and the National Basic Research Program of China (973 Program, no. 2014CB541600 to Hua Feng).

\section{References}

[1] T. T. Hien, N. J. White, and White, "Qinghaosu," The Lancet, vol. 341, no. 8845, pp. 603-608, 1993.

[2] E. Pelfrene, M.-H. Pinheiro, and M. Cavaleri, "Artemisininbased combination therapy in the treatment of uncomplicated malaria: review of recent regulatory experience at the European Medicines Agency," International Health, vol. 7, no. 4, pp. 239246, 2015.

[3] WHO, Guidelines for the Treatment of Malaria, WHO, Geneva, Switzerland, 3rd edition, 2015.

[4] K. Zhao and Z. Song, "Distribution and excretion of artesunate in rats," Proceedings of the Chinese Academy of Medical Sciences and the Peking Union Medical College, vol. 4, no. 4, pp. 186-188, 1989.

[5] H. J. Woerdenbag, T. A. Moskal, N. Pras et al., "Cytotoxicity of artemisinin-related endoperoxides to Ehrlich ascites tumor cells," Journal of Natural Products, vol. 56, no. 6, pp. 849-856, 1993.

[6] G.-Q. Zheng, "Cytotoxic terpenoids and flavonoids from Artemisia annua," Planta Medica, vol. 60, no. 1, pp. 54-57, 1994.

[7] L. Lu, "Study on effect of Cordyceps sinensis and artemisinin in preventing recurrence of lupus nephritis," Chinese Journal of Integrated Traditional and Western Medicine, vol. 22, no. 3, pp. 169-171, 2002.

[8] S. Jauréguiberry, M. Thellier, P. A. Ndour et al., "Delayedonset hemolytic anemia in patients with travel-associated severe malaria treated with Artesunate, France, 2011-2013," Emerging Infectious Diseases, vol. 21, no. 5, pp. 804-812, 2015.

[9] J. Gómez-Junyent, P. Ruiz-Panales, A. Calvo-Cano, J. Gascón, and J. Muñoz, "Delayed haemolysis after artesunate therapy in a cohort of patients with severe imported malaria due to Plasmodium falciparum," Enfermedades Infecciosas y Microbiología Clínica, 2015.

[10] F. B. Li and H. Z. Pan, "Peroxidative antimalaria mechanism of sodium artesunate," Acta Academiae Medicinae Sinicae, vol. 11, no. 3, pp. 180-184, 1989.
[11] Y. I. Zhao, W. K. Hanton, and K.-H. Lee, "Antimalarial agents, 2. Artesunate, an inhibitor of cytochrome oxidase activity in Plasmodium berghei," Journal of Natural Products, vol. 49, no. 1, pp. 139-142, 1986.

[12] N. Berdelle, T. Nikolova, S. Quiros, T. Efferth, and B. Kaina, "Artesunate induces oxidative DNA damage, sustained DNA double-strand breaks, and the ATM/ATR damage response in cancer cells," Molecular Cancer Therapeutics, vol. 10, no. 12, pp. 2224-2233, 2011.

[13] T. Efferth and F. Oesch, "Oxidative stress response of tumor cells: microarray-based comparison between artemisinins and anthracyclines," Biochemical Pharmacology, vol. 68, no. 1, pp. 3$10,2004$.

[14] Q. Wang, L.-M. Wu, Y. Zhao, X.-L. Zhang, and N.-P. Wang, "The anticancer effect of artesunate and its mechanism," Acta Pharmaceutica Sinica, vol. 37, no. 6, pp. 477-478, 2002.

[15] S. Reichert, V. Reinboldt, S. Hehlgans, T. Efferth, C. Rödel, and F. Rödel, "A radiosensitizing effect of artesunate in glioblastoma cells is associated with a diminished expression of the inhibitor of apoptosis protein survivin," Radiotherapy and Oncology, vol. 103, no. 3, pp. 394-401, 2012.

[16] E. Ooko, M. E. M. Saeed, O. Kadioglu et al., "Artemisinin derivatives induce iron-dependent cell death (ferroptosis) in tumor cells," Phytomedicine, vol. 22, no. 11, pp. 1045-1054, 2015.

[17] W. Jiang, B. Li, X. Zheng et al., "Artesunate in combination with oxacillin protect sepsis model mice challenged with lethal live methicillin-resistant Staphylococcus aureus (MRSA) via its inhibition on proinflammatory cytokines release and enhancement on antibacterial activity of oxacillin," International Immunopharmacology, vol. 11, no. 8, pp. 1065-1073, 2011.

[18] M. Alenquer, S. Tenreiro, and I. Sá-Correia, "Adaptive response to the antimalarial drug artesunate in yeast involves Pdrlp/Pdr3p-mediated transcriptional activation of the resistance determinants TPO1 and PDR5," FEMS Yeast Research, vol. 6, no. 8, pp. 1130-1139, 2006.

[19] B. Li, J. Li, X. Pan et al., "Artesunate protects sepsis model mice challenged with Staphylococcus aureus by decreasing TNF- $\alpha$ release via inhibition TLR2 and Nod 2 mRNA expressions and transcription factor NF- $\kappa \mathrm{B}$ activation," International Immunopharmacology, vol. 10, no. 3, pp. 344-350, 2010.

[20] C. Canivet, R. Menasria, C. Rhéaume, J. Piret, and G. Boivin, "Valacyclovir combined with artesunate or rapamycin improves the outcome of herpes simplex virus encephalitis in mice compared to antiviral therapy alone," Antiviral Research, vol. 123, pp. 105-113, 2015.

[21] L. A. El Zawawy, "Effect of artesunate on Toxoplasma gondii: in vitro and in vivo studies," Journal of the Egyptian Society of Parasitology, vol. 38, no. 1, pp. 185-201, 2008.

[22] M. E. Sarciron, C. Saccharin, A. F. Petavy, and F. Peyron, "Effects of artesunate, dihydroartemisinin, and an artesunatedihydroartemisinin combination against Toxoplasma gondii," American Journal of Tropical Medicine and Hygiene, vol. 62, no. 1, pp. 73-76, 2000.

[23] W. J. Le, J. Q. You, and J. Y. Mei, "Chemotherapeutic effect of artesunate in experimental schistosomiasis," Acta Pharmaceutica Sinica, vol. 18, no. 8, pp. 619-621, 1983.

[24] R. Liu, H.-F. Dong, Y. Guo, Q.-P. Zhao, and M.-S. Jiang, “Efficacy of praziquantel and artemisinin derivatives for the treatment and prevention of human schistosomiasis: a systematic review and meta-analysis," Parasites and Vectors, vol. 4, no. 1, article no. 201, 2011. 
[25] M. Spicher, C. Roethlisberger, C. Lany et al., "In vitro and in vivo treatments of Echinococcus protoscoleces and metacestodes with artemisinin and artemisinin derivatives," Antimicrobial Agents and Chemotherapy, vol. 52, no. 9, pp. 3447-3450, 2008.

[26] S. Zuo, H. Ge, Q. Li et al., "Artesunate protected blood-brain barrier via sphingosine 1 phosphate receptor $1 /$ phosphatidylinositol 3 kinase pathway after subarachnoid hemorrhage in rats," Molecular Neurobiology, 2016.

[27] U. P. Okorji and O. A. Olajide, "A semi-synthetic derivative of artemisinin, artesunate inhibits prostaglandin $\mathrm{E} 2$ production in LPS/IFN $\gamma$-activated BV2 microglia," Bioorganic and Medicinal Chemistry, vol. 22, no. 17, pp. 4726-4734, 2014.

[28] I.-S. Lee, D.-K. Ryu, J. Lim, S. Cho, B. Y. Kang, and H. J. Choi, "Artesunate activates Nrf2 pathway-driven anti-inflammatory potential through ERK signaling in microglial BV2 cells," Neuroscience Letters, vol. 509, no. 1, pp. 17-21, 2012.

[29] U. C. Schneider, A.-M. Davids, S. Brandenburg et al., "Microglia inflict delayed brain injury after subarachnoid hemorrhage," Acta Neuropathologica, vol. 130, no. 2, pp. 215-231, 2015.

[30] Z. Zhou and Y. Feng, "Artesunate reduces proliferation, interferes DNA replication and cell cycle and enhances apoptosis in vascular smooth muscle cells," Journal of Huazhong University of Science and Technology Medical Sciences, vol. 25, no. 2, pp. 135-136, 2005.

[31] C. O. Borel, A. McKee, A. Parra et al., "Possible role for vascular cell proliferation in cerebral vasospasm after subarachnoid hemorrhage," Stroke, vol. 34, no. 2, pp. 427-433, 2003.

[32] S. Chen, H. Feng, P. Sherchan et al., "Controversies and evolving new mechanisms in subarachnoid hemorrhage," Progress in Neurobiology, vol. 115, pp. 64-91, 2014.

[33] L. Clemmer, Y. C. Martins, G. M. Zanini, J. A. Frangos, and L. J. M. Carvalho, "Artemether and artesunate show the highest efficacies in rescuing mice with late-stage cerebral malaria and rapidly decrease leukocyte accumulation in the brain," Antimicrobial Agents and Chemotherapy, vol. 55, no. 4, pp. 13831390, 2011.

[34] M. Chen, M. Kappel, M. Lemnge, I. C. Bygbjerg, T. G. Theander, and A. Kharazmi, "In vitro effects of artesunate and other antimalarial agents on the function of human lymphocytes and neutrophils," Transplantation Proceedings, vol. 26, no. 6, pp. 3172-3174, 1994.

[35] V. B. Konkimalla, M. Blunder, B. Korn et al., "Effect of artemisinins and other endoperoxides on nitric oxide-related signaling pathway in RAW 264.7 mouse macrophage cells," Nitric Oxide: Biology and Chemistry, vol. 19, no. 2, pp. 184-191, 2008.

[36] T. C. Mota, P. C. S. Cardoso, L. M. Gomes et al., "In vitro evaluation of the genotoxic and cytotoxic effects of artesunate, an antimalarial drug, in human lymphocytes," Environmental and Molecular Mutagenesis, vol. 52, no. 7, pp. 590-594, 2011.

[37] L. Yang, J. R. Kowalski, X. Zhan, S. M. Thomas, and F. W. Luscinskas, "Endothelial cell cortactin phosphorylation by $\mathrm{Src}$ contributes to polymorphonuclear leukocyte transmigration in vitro," Circulation Research, vol. 98, no. 3, pp. 394-402, 2006.

[38] Y. Kubo, K. Ogasawara, S. Kakino et al., "Serum inflammatory adhesion molecules and high-sensitivity C-reactive protein correlates with delayed ischemic neurologic deficits after subarachnoid hemorrhage," Surgical Neurology, vol. 69, no. 6, pp. 592-596, 2008.

[39] M. Bavbek, R. Polin, A.-L. Kwan, A. S. Arthur, N. F. Kassell, and K. S. Lee, "Monoclonal antibodies against ICAM-1 and CD18 attenuate cerebral vasospasm after experimental subarachnoid hemorrhage in rabbits," Stroke, vol. 29, no. 9, pp. 1930-1935, 1998.

[40] M. C. Souza, F. H. M. Paixão, F. K. Ferraris, I. Ribeiro, and M. D. G. M. O. Henriques, "Artesunate exerts a direct effect on endothelial cell activation and NF- $\kappa \mathrm{B}$ translocation in a mechanism independent of plasmodium killing," Malaria Research and Treatment, vol. 2012, Article ID 679090, 12 pages, 2012.

[41] A. Gruber, A. Reinprecht, U. M. Illievich et al., "Extracerebral organ dysfunction and neurologic outcome after aneurysmal subarachnoid hemorrhage," Critical Care Medicine, vol. 27, no. 3, pp. 505-514, 1999.

[42] W. J. Schuiling, P. J. W. Dennesen, and G. J. E. Rinkel, "Extracerebral organ dysfunction in the acute stage after aneurysmal subarachnoid hemorrhage," Neurocritical Care, vol. 3, no. 1, pp. 1-10, 2005.

[43] S. Radolf, N. Smoll, C. Drenckhahn, J. P. Dreier, P. Vajkoczy, and A. S. Sarrafzadeh, "Cerebral lactate correlates with early onset pneumonia after aneurysmal SAH," Translational Stroke Research, vol. 5, no. 2, pp. 278-285, 2014.

[44] P. M. Cobelens, I. A. C. W. Tiebosch, R. M. Dijkhuizen et al., "Interferon- $\beta$ attenuates lung inflammation following experimental subarachnoid hemorrhage," Critical Care, vol. 14, article R157, 2010.

[45] C. Gera and J. Dhanoa, "Vivax-induced ARDS: report of two cases," Journal of Association of Physicians of India, vol. 58, pp. 48-50, 2010.

[46] W. E. Ho, C. Cheng, H. Y. Peh et al., "Anti-malarial drug artesunate ameliorates oxidative lung damage in experimental allergic asthma," Free Radical Biology and Medicine, vol. 53, no. 3, pp. 498-507, 2012.

[47] D. S. W. Ng, W. Liao, W. S. D. Tan, T. K. Chan, X. Y. Loh, and W. S. F. Wong, "Anti-malarial drug artesunate protects against cigarette smoke-induced lung injury in mice," Phytomedicine, vol. 21, no. 12, pp. 1638-1644, 2014.

[48] C. J. van Asch, M. J. Luitse, G. J. Rinkel, I. van der Tweel, A. Algra, and C. J. Klijn, "Incidence, case fatality, and functional outcome of intracerebral haemorrhage over time, according to age, sex, and ethnic origin: a systematic review and metaanalysis," The Lancet Neurology, vol. 9, no. 2, pp. 167-176, 2010.

[49] R. F. Keep, Y. Hua, and G. Xi, "Intracerebral haemorrhage: mechanisms of injury and therapeutic targets," The Lancet Neurology, vol. 11, no. 8, pp. 720-731, 2012.

[50] J. Wang, J. Fields, C. Zhao et al., "Role of Nrf2 in protection against intracerebral hemorrhage injury in mice," Free Radical Biology \& Medicine, vol. 43, no. 3, pp. 408-414, 2007.

[51] Y. He, S. Wan, Y. Hua, R. F. Keep, and G. Xi, "Autophagy after experimental intracerebral hemorrhage," Journal of Cerebral Blood Flow and Metabolism, vol. 28, no. 5, pp. 897-905, 2008.

[52] S. Hu, G. Xi, H. Jin, Y. He, R. F. Keep, and Y. Hua, "Thrombininduced autophagy: a potential role in intracerebral hemorrhage," Brain Research, vol. 1424, pp. 60-66, 2011.

[53] B. Yuan, H. Shen, L. Lin, T. Su, L. Zhong, and Z. Yang, "Autophagy promotes microglia activation through beclin-1Atg5 pathway in intracerebral hemorrhage," Molecular Neurobiology, 2016.

[54] R. W. Button, F. Lin, E. Ercolano et al., "Artesunate induces necrotic cell death in schwannoma cells," Cell Death \& Disease, vol. 5, no. 10, Article ID e1466, 2014.

[55] A. Hamacher-Brady, H. A. Stein, S. Turschner et al., "Artesunate activates mitochondrial apoptosis in breast cancer cells via 
iron-catalyzed lysosomal reactive oxygen species production," Journal of Biological Chemistry, vol. 286, no. 8, pp. 6587-6601, 2011.

[56] A. M. Gopalakrishnan and N. Kumar, "Antimalarial action of artesunate involves DNA damage mediated by reactive oxygen species," Antimicrobial Agents and Chemotherapy, vol. 59, no. 1, pp. 317-325, 2015.

[57] K. Chen, L.-M. Shou, F. Lin et al., "Artesunate induces G2/M cell cycle arrest through autophagy induction in breast cancer cells," Anti-Cancer Drugs, vol. 25, no. 6, pp. 652-662, 2014.

[58] Y.-C. Wang, S. Lin, and Q.-W. Yang, “Toll-like receptors in cerebral ischemic inflammatory injury," Journal of Neuroinflammation, vol. 8, article 134, 2011.

[59] D. Wang, J. Shi, S. Lv et al., "Artesunate attenuates lipopolysaccharide-stimulated proinflammatory responses by suppressing TLR4, MyD88 expression, and NF- $\kappa$ B activation in microglial cells," Inflammation, vol. 38, no. 5, pp. 1925-1932, 2015.

[60] P. Zhang, X.-M. Chen, B.-D. Luo et al., "Effects of artesunate on CD14 and toll-like receptor 4 in peritoneal macrophages of mice with heat stroke endotoxemia," Chinese Journal of Industrial Hygiene and Occupational Diseases, vol. 24, no. 4, pp. 226-228, 2006.

[61] L. Lai, Y. Chen, X. Tian et al., "Artesunate alleviates hepatic fibrosis induced by multiple pathogenic factors and inflammation through the inhibition of LPS/TLR4/NF- $\kappa$ B signaling pathway in rats," European Journal of Pharmacology, vol. 765, pp. 234-241, 2015.

[62] R. Schmid-Elsaesser, E. Hungerhuber, S. Zausinger, A. Baethmann, and H.-J. Reulen, "Neuroprotective efficacy of combination therapy with two different antioxidants in rats subjected to transient focal ischemia," Brain Research, vol. 816, no. 2, pp. 471479, 1999.

[63] J. Álvarez-Sabín, O. Maisterra, E. Santamarina, and C. S. Kase, "Factors influencing haemorrhagic transformation in ischaemic stroke," The Lancet Neurology, vol. 12, no. 7, pp. 689-705, 2013.

[64] G. F. Hamann, Y. Okada, and G. J. Del Zoppo, "Hemorrhagic transformation and microvascular integrity during focal cerebral ischemia/reperfusion," Journal of Cerebral Blood Flow and Metabolism, vol. 16, no. 6, pp. 1373-1378, 1996.

[65] Y. Gasche, J.-C. Copin, T. Sugawara, M. Fujimura, and P. H. Chan, "Matrix metalloproteinase inhibition prevents oxidative stress-associated blood-brain barrier disruption after transient focal cerebral ischemia," Journal of Cerebral Blood Flow and Metabolism, vol. 21, no. 12, pp. 1393-1400, 2001.

[66] Y. Xu, W. Liu, B. Fang, S. Gao, and J. Yan, "Artesunate ameliorates hepatic fibrosis induced by bovine serum albumin in rats through regulating matrix metalloproteinases," European Journal of Pharmacology, vol. 744, pp. 1-9, 2014.

[67] D. Magenta, E. Sangiovanni, N. Basilico et al., "Inhibition of metalloproteinase- 9 secretion and gene expression by artemisinin derivatives," Acta Tropica, vol. 140, pp. 77-83, 2014.

[68] M. J. A. Luitse, G. J. Biessels, G. E. H. M. Rutten, and L. J. Kappelle, "Diabetes, hyperglycaemia, and acute ischaemic stroke," The Lancet Neurology, vol. 11, no. 3, pp. 261-271, 2012.

[69] L. Yu, J. F. Chen, X. Shuai et al., "Artesunate protects pancreatic beta cells against cytokine-induced damage via SIRT1 inhibiting NF- $\kappa$ B activation," Journal of Endocrinological Investigation, vol. 39, no. 1, pp. 83-91, 2016.

[70] K. Ritz, N. P. Denswil, O. C. G. Stam, J. J. Van Lieshout, and M. J. A. P. Daemen, "Cause and mechanisms of intracranial atherosclerosis," Circulation, vol. 130, no. 16, pp. 1407-1414, 2014.
[71] Y. L. Wang, Z. J. Wang, H. L. Shen, M. Yin, and K. X. Tang, "Effects of artesunate and ursolic acid on hyperlipidemia and its complications in rabbit," European Journal of Pharmaceutical Sciences, vol. 50, no. 3-4, pp. 366-371, 2013.

[72] Y. Wang, Z. Wang, H. Shen, M. Yin, and K. Tang, "The hypolipidemic effect of artesunate and ursolic acid in rats," Pakistan Journal of Pharmaceutical Sciences, vol. 28, no. 3, pp. 871-874, 2015.

[73] R. T. Merrell, "Brain tumors," Disease-a-Month, vol. 58, no. 12, pp. 678-689, 2012.

[74] G. Karpel-Massler, M.-A. Westhoff, R. E. Kast et al., "Artesunate enhances the antiproliferative effect of temozolomide on U87MG and A172 glioblastoma cell lines," Anti-Cancer Agents in Medicinal Chemistry, vol. 14, no. 2, pp. 313-318, 2014.

[75] R. E. Kast, J. A. Boockvar, A. Brüning et al., "A conceptually new treatment approach for relapsed glioblastoma: coordinated undermining of survival paths with nine repurposed drugs (CUSP9) by the International Initiative for Accelerated Improvement of Glioblastoma Care," Oncotarget, vol. 4, no. 4, pp. 502-530, 2013.

[76] T. Efferth, T. Ramirez, E. Gebhart, and M.-E. Halatsch, "Combination treatment of glioblastoma multiforme cell lines with the anti-malarial artesunate and the epidermal growth factor receptor tyrosine kinase inhibitor OSI-774," Biochemical Pharmacology, vol. 67, no. 9, pp. 1689-1700, 2004.

[77] M. Czarnecka, E. Trinh, C. Lu et al., "Neuropeptide y receptor $\mathrm{Y} 5$ as an inducible pro-survival factor in neuroblastoma: Implications for tumor chemoresistance," Oncogene, vol. 34, no. 24, pp. 3131-3143, 2015.

[78] M. Michaelis, M. C. Kleinschmidt, S. Barth et al., "Anti-cancer effects of artesunate in a panel of chemoresistant neuroblastoma cell lines," Biochemical Pharmacology, vol. 79, no. 2, pp. 130-136, 2010.

[79] Z.-G. Mao, J. Zhou, H. Wang et al., "Artesunate inhibits cell proliferation and decreases growth hormone synthesis and secretion in GH3 cells," Molecular Biology Reports, vol. 39, no. 5, pp. 6227-6234, 2012.

[80] R. W. Button, F. Lin, E. Ercolano et al., "Artesunate induces necrotic cell death in schwannoma cells," Cell Death and Disease, vol. 5, no. 10, Article ID e1466, 2014.

[81] D. Kobayashi, M. Hirayama, Y. Komohara et al., "Translationally controlled tumor protein is a novel biological target for neurofibromatosis type 1-associated tumors," Journal of Biological Chemistry, vol. 289, no. 38, pp. 26314-26326, 2014.

[82] C. Zhou, W. Pan, X. P. Wang, and T. S. Chen, "Artesunate induces apoptosis via a Bak-mediated caspase-independent intrinsic pathway in human lung adenocarcinoma cells," Journal of Cellular Physiology, vol. 227, no. 12, pp. 3778-3786, 2012.

[83] Z.-Y. Zhang, S.-Q. Yu, L.-Y. Miao et al., "Artesunate combined with vinorelbine plus cisplatin in treatment of advanced nonsmall cell lung cancer: a randomized controlled trial," Journal of Chinese Integrative Medicine, vol. 6, no. 2, pp. 134-138, 2008.

[84] S. A. K. Rasheed, T. Efferth, I. A. Asangani, and H. Allgayer, "First evidence that the antimalarial drug artesunate inhibits invasion and in vivo metastasis in lung cancer by targeting essential extracellular proteases," International Journal of Cancer, vol. 127, no. 6, pp. 1475-1485, 2010.

[85] X. Chen, K. Han, F. Chen, C. Wu, and W. Huang, "Effects of artesunate on the invasion of lung adenocarcinoma A549 cells and expression of ICAM-1 and MMP-9," Chinese Journal of Lung Cancer, vol. 16, no. 11, pp. 567-571, 2013. 
[86] H.-Y. Dong and Z.-F. Wang, "Antitumor effects of artesunate on human breast carcinoma MCF-7 cells and IGF-IR expression in nude mice xenografts," Chinese Journal of Cancer Research, vol. 26, no. 2, pp. 200-207, 2014.

[87] E. Seo, B. Wiench, R. Hamm et al., "Cytotoxicity of natural products and derivatives toward MCF-7 cell monolayers and cancer stem-like mammospheres," Phytomedicine, vol. 22, no. 4, pp. 438-443, 2015.

[88] Q. Li, W. Wang, Y. Liu et al., “The biological characteristics of a novel camptothecin-artesunate conjugate," Bioorganic and Medicinal Chemistry Letters, vol. 25, no. 1, pp. 148-152, 2015.

[89] W. M. Liu, A. M. Gravett, and A. G. Dalgleish, "The antimalarial agent artesunate possesses anticancer properties that can be enhanced by combination strategies," International Journal of Cancer, vol. 128, no. 6, pp. 1471-1480, 2011.

[90] L. Hou, K. E. Block, and H. Huang, "Artesunate abolishes germinal center B cells and inhibits autoimmune arthritis," PLoS ONE, vol. 9, no. 8, Article ID e104762, 2014.

[91] O. Jin, H. Zhang, Z. Gu et al., "A pilot study of the therapeutic efficacy and mechanism of artesunate in the MRL/lpr murine model of systemic lupus erythematosus," Cellular and Molecular Immunology, vol. 6, no. 6, pp. 461-467, 2009.

[92] S. Mani, S. S. Mondal, G. Guha et al., "Acute disseminated encephalomyelitis after mixed malaria infection (plasmodium falciparum and plasmodium vivax) with MRI closely simulating multiple sclerosis," Neurologist, vol. 17, no. 5, pp. 276-278, 2011.

[93] J.-Q. Shi, C.-C. Zhang, X.-L. Sun et al., "Antimalarial drug artemisinin extenuates amyloidogenesis and neuroinflammation in APPswe/PS1dE9 transgenic mice via inhibition of nuclear factor- $\kappa \mathrm{B}$ and NLRP3 inflammasome activation," CNS Neuroscience \& Therapeutics, vol. 19, no. 4, pp. 262-268, 2013.

[94] M. Bartkovjak, R. Ianetti, K. Kutna et al., "Cerebral malaria in children in South Sudan: 8 years experience in 261 cases," Neuroendocrinology Letters, vol. 28, no. 2, pp. 45-46, 2007.

[95] A. M. Dondorp, C. I. Fanello, I. C. Hendriksen et al., "Artesunate versus quinine in the treatment of severe falciparum malaria in African children (AQUAMAT): an open-label, randomised trial," The Lancet, vol. 376, no. 9753, pp. 1647-1657, 2010.

[96] C. Mwita, L. Mwai, and C. Newton, "Antiepileptic properties of quinine: a systematic review," Annals of Neurosciences, vol. 19, no. 1, pp. 14-20, 2012.

[97] S. Parikh, M. Koch, and R. K. Narayan, "Traumatic brain injury," International Anesthesiology Clinics, vol. 45, no. 3, pp. 119-135, 2007.

[98] A. Mbengue, S. Bhattacharjee, T. Pandharkar et al., "A molecular mechanism of artemisinin resistance in Plasmodium falciparum malaria," Nature, vol. 520, no. 7549, pp. 683-687, 2015.

[99] C. Cheng, W. E. Ho, F. Y. Goh et al., "Anti-malarial drug artesunate attenuates experimental allergic asthma via inhibition of the phosphoinositide 3-kinase/Akt pathway," PLoS ONE, vol. 6, no. 6, Article ID e20932, 2011.

[100] O. Thanaketpaisarn, P. Waiwut, H. Sakurai, and I. Saiki, "Artesunate enhances TRAIL-induced apoptosis in human cervical carcinoma cells through inhibition of the NF- $\kappa$ B and PI3K/Akt signaling pathways," International Journal of Oncology, vol. 39, no. 1, pp. 279-285, 2011. 


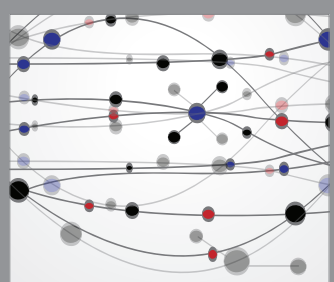

The Scientific World Journal
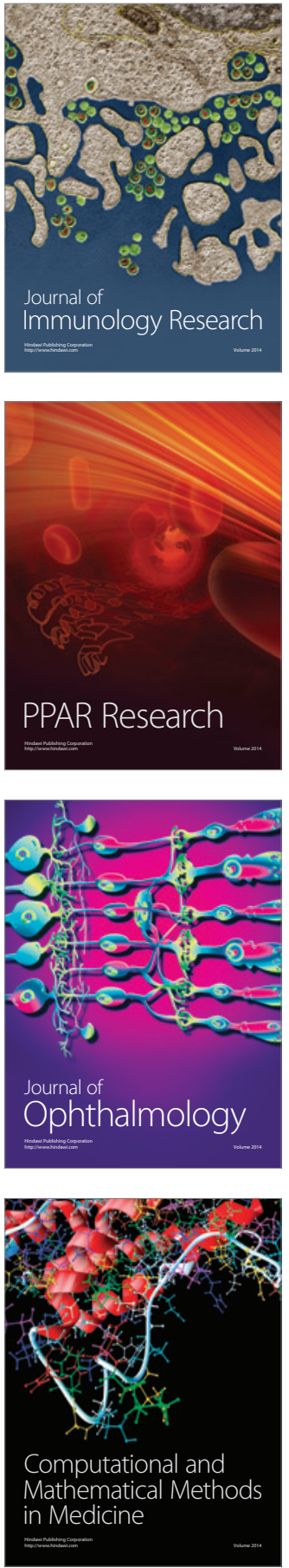

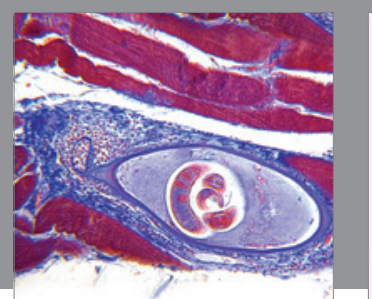

Gastroenterology Research and Practice

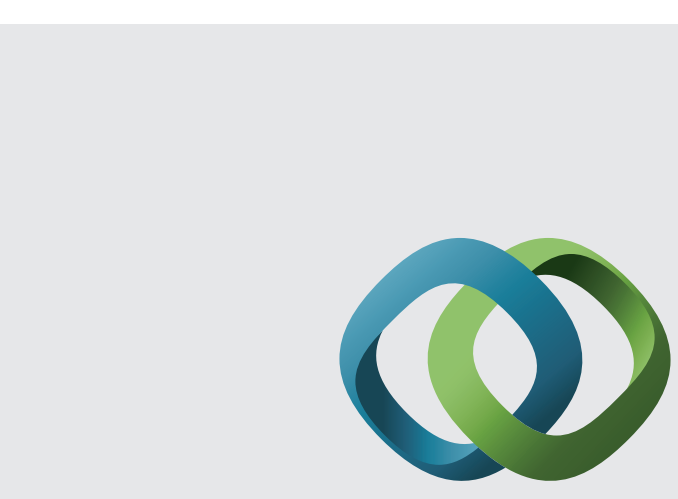

\section{Hindawi}

Submit your manuscripts at

http://www.hindawi.com
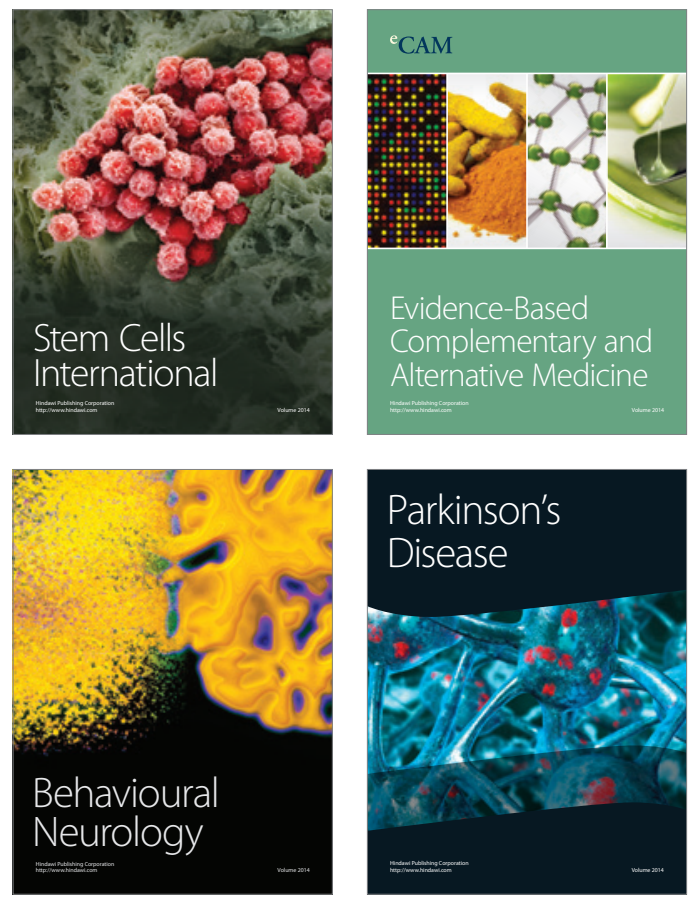
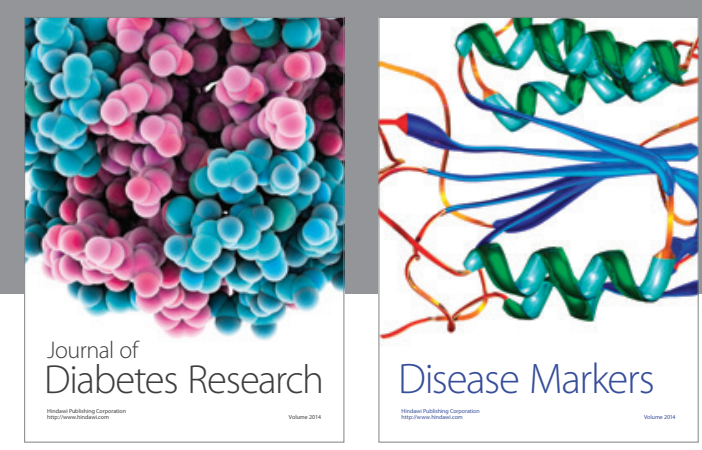

Disease Markers
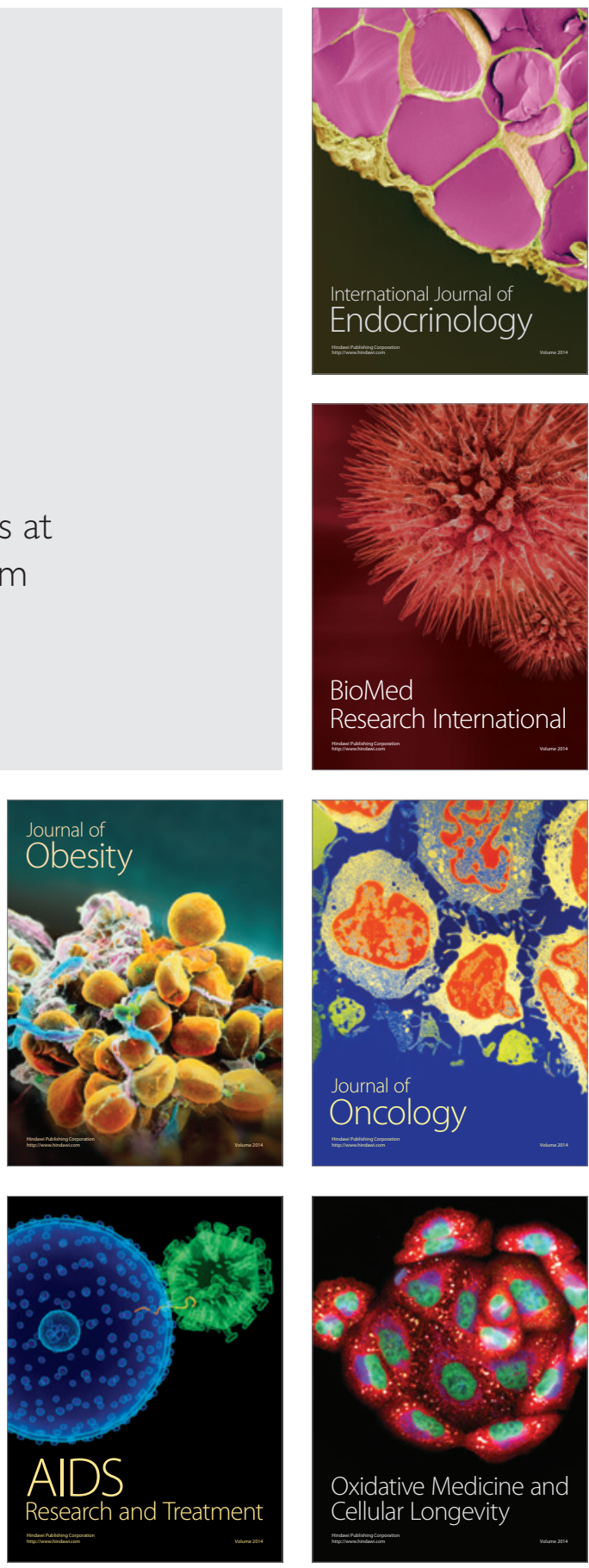